\title{
Benefits of Vehicle Automation for Public Transportation Operations
}

\author{
Kareem Othman ${ }^{1,2 *}$ \\ ${ }^{1}$ Civil engineering department, University of Toronto, Toronto, Canada \\ ${ }^{2}$ Public works department, Faculty of engineering, Cairo University, Giza, Egypt
}

*Corresponding author: Kareem Othman, Civil engineering department, University of Toronto, Toronto, Ontario, Canada; Public works department, Faculty of engineering, Cairo University, Giza, Egypt.

\begin{abstract}
Over the past few years, vehicle automation has witnessed tremendous focus from both the academic perspective and industrial perspective. However, autonomous cars have been the main focus of many studies in the literature, unlike research on the automation of buses which is rare. Thus, this paper discusses the benefits of the application of the automation technology to the public transportation service. Autonomous buses can improve the quality of transit service, reduce the operating costs dramatically, increase vehicle utilization, increase the frequency of the transit service with low operating costs and allow for vehicle platooning. While many benefits can be achieved from the automation of buses, further research studies and focus from the academia are required to quantify these benefits, estimate the implications of the automation of public transportation service, and understand how these implications will affect both transit agencies and transit users.
\end{abstract}

Keywords: Autonomous vehicles; Autonomous buses; Public transportation

\section{Introduction}

Over the last few years, media have been fascinated by autonomous vehicles (AVs) and their implications in our lives. It is expected that AVs have the potential to provide many benefits such as:

1. Safety: it is estimated that human error contributes to $90 \%$ of traffic accidents that mainly result from distraction of drivers. Thus, AVs have the potential to reduce the collision rate $[1,2]$.

2. Increase lane capacity: due to the high level of communication between AVs, it is expected that AVs can operate with much shorter lateral and headway gap. Additionally, this high level of communication allows AVs to travel in platoons. As a result, AVs have the potential to increase lane capacity $[3,4]$.

3. Accessibility: AVs have the potential to improve the accessibility for people with limited transportation such as old people and disabled [5-9].

On the other hand, the main question is how AVs will operate as fleets and what are their impact on public transportation as one of the main advantages of public transportation is the ability to move a large number of people with much fewer number of vehicles to solve congestion issues and pollution challenges. There are growing consensus that AVs might worsen traffic and environmental conditions even if AVs use electric engines. AVs are expected to increase the vehicle kilometers travelled (VKT) significantly. Passengers will be able to participate in other activities while travelling, which will motivate people to create longer trips [10]. For example, some people might choose to live further away from their work location (for example to pay lower rent) and spend longer trips while spending their work trip time sleeping. 
Additionally, AVs will motivate people to create additional trips. As a result, AVs might worsen traffic conditions and increase congestion levels [11]. Thus, researchers at ETH Zurich have suggested that the introduction of AVs might not be environmentally friendly and that cities might need to prohibit the use of personal AVs [12] and invest more in autonomous buses. This paper illustrates the benefits of the application of vehicle automation technology in the public transportation sector.

\section{Potential benefits of Autonomous Buses (ABs)}

1. Transit service will be much standardized as there are no different driving styles, good and bad drivers. Thus, Driver behaviors will not be a factor that influences reliability, and speed.

2. ABs have the potential to reduce the operating costs because of the elimination of the wage cost of bus drivers (which represent almost 50\% of the overall operating costs). Additionally, the fuel cost will be reduced significantly as according to today's trends that autonomous vehicles usually have electric engines. A financial analysis by Nagy \& Horváth [13] show that ABs can reduce the overall costs (operating and capital costs) by $17-24 \%$ compared to the current system. This reduction in the operating costs will reduce transit fare significantly and attract more people to transit.

3. ABs have the potential to increase the vehicle utilization. In human driven buses, $\mathrm{ABs}$ and drivers are connected. If the driver takes the rest time, then the vehicle (which is the most expensive component of the system) rests too. Thus, the vehicle utilization does not exceed 60 or $70 \%$, which in turn means that the vehicle does not generate income in third part of its working time because of the rest time. This utilization can be increased in autonomous buses. For example, a study by Nagy \& Horváth [13] in the city of Eger, Hungry shows that ABs have the potential to reduce the required fleet from 37 buses to 32 buses (saving 4 buses) to provide the same service. This reduction in the fleet size, means lower operating costs.

4. ABs have the potential to increase the frequency without significant increase in the overall costs. Generally, passengers preferer short headways to reduce their waiting time, while Operators preferer long headways to reduce their operating costs and fleet size. Thus, estimating the service frequency is an optimization problem that balances between the operating costs and passenger costs. ABs will be a game changer that will allow for the use of smaller buses and with much lower operating costs (No driver cost, no fuel cost) compared to human driven buses. Thus, ABs will allow for providing higher frequency with much lower operating costs. This will be very helpful for people in rural or devastated areas. This increase in the frequency will reduce passengers waiting time and will attract more users to transit. Figure 1 shows the difference in the operating costs between the case of human driven buses and ABs (Figure 1).

5. ABs have the potential to improve the bus rapid transit (BRT) service because of the ability of buses to form platoons. BRT gives buses many of the best qualities of trains without the accompanying price tag. On the other hand, light rail transit (LRT) improves mobility along their communities' busiest corridors. The main advantage for adapting LRT is the high-level of capacity offered for high demand routes. However, the main issue associate with LRT systems is the high construction cost. One of the major advantages of bus automation is that it will allow buses to travel in platoons and formulate a system that has much higher capacity similar to the LRT system (as shown in Figure 2) but with much lower capital cost(as the cost of rail construction is eliminated). Autonomous vehicle technology would allow BRT operators to link multiple buses together seamlessly, adapting to demand and adding capacity as needed (Figure 2).

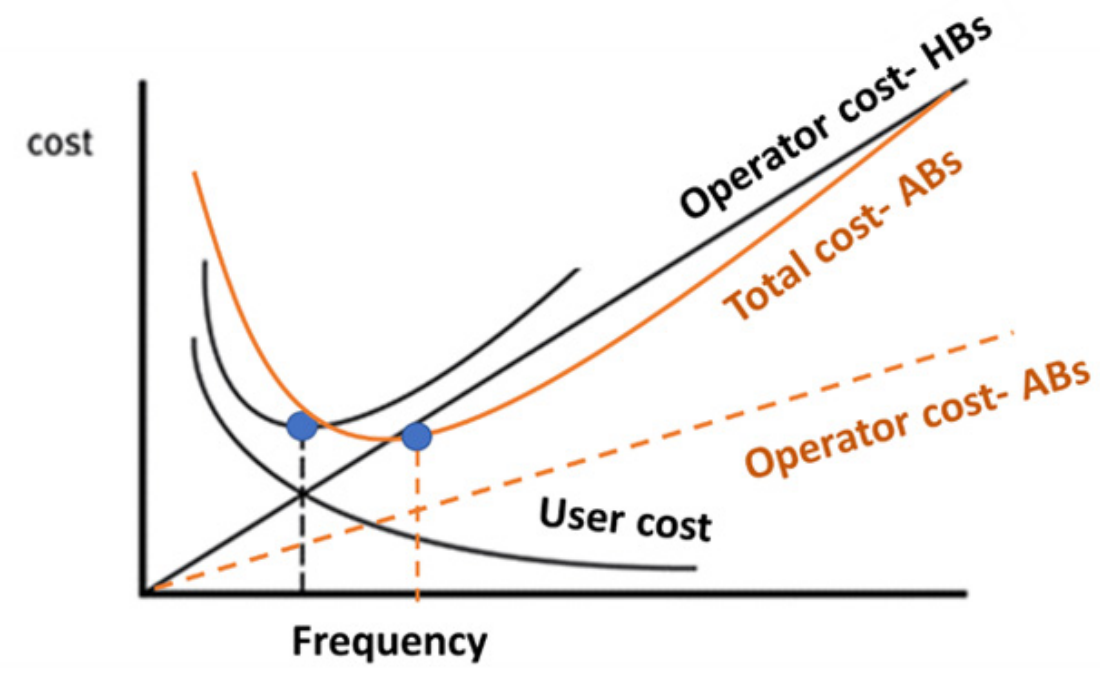

Figure 1: Relation between frequency and costs for the case of human driven buses and ABs (HBs= Human-driven buses; $A B s=$ Autonomous buses). 

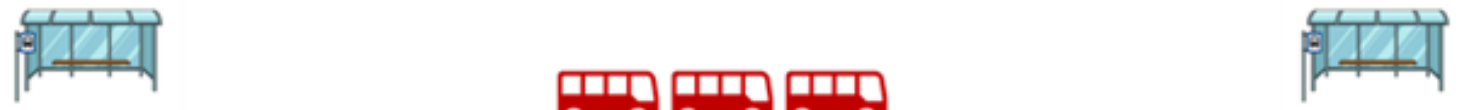

Figure 2: A platoon of Autonomous Buses.

\section{Dynamic Transit lanes (DTL)}

Exclusive bus lanes (EBL) or sometimes called conventional transit lanes, which cars are not allowed to use, have been implemented in many urban areas across the world with the goal of improving transit speed. On the other hand, the major issue for the exclusive bus lanes is the significant reduction in the road capacity for other road users. Transit usually runs infrequently, leaving large gaps during which the EBL are not used. Thus, the idea of the intermittent bus lanes (IBL) was proposed in 2001 by Viegas and Lu. IBLs allow private vehicles to use transit lanes when transit is not present $[14,15]$. IBLs are accessible for cars at specific times depending on the transit schedules and this accessibility was communicated through variable message signs or flashlights [16]. IBLs were implemented in two cases studies one in Melbourne, Australia and the other one in Lisbon, Portugal. Results of these two case studies showed significant improvement in transit speed (10-25\%). However, previous studies highlighted several issues preventing the efficient use of DTLs. For example, communication changes in transit lanes need to be communicated quickly to drivers. However, conditions that increase the congestion level, such as the adverse weather or the peak hour demand, might reduce the visibility of the variable message signs or the flashlights [17]. These issues can be addressed by connected and autonomous vehicles technology that is entirely controlled by computers and does not need the driver's intervention. In 2018, Levin and Khani [16] proposed the dynamic transit lanes idea that employees vehicle connectivity and automation to change the accessibility of the transit lane at small spatial and temporal intervals. DTLs are lanes that are accessible to cars when transit is not present. When the bus travel along the DTL, private vehicles in front of the bus exit the lane, and private vehicles behind the bus can move into the lane once the bus has passed as described in Figure 3. Numerical results for the application of the DTL for the city of Austin show that that both transit and general traffic would experience significant benefits. For examples, results show that the dynamic transit lanes if applied with transit signal priority allow transit to move nearly at free flow on the corridor despite congestion (Figure 3).
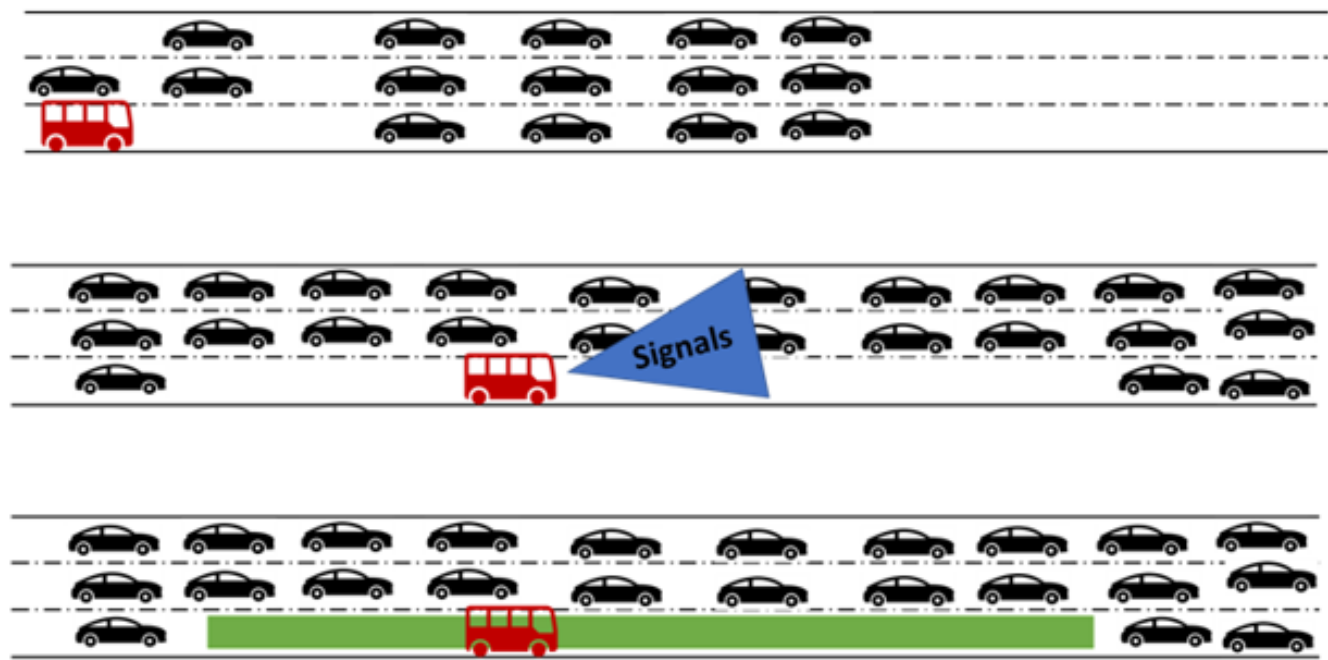

Figure 3: Dynamic transit lane.

\section{Speed Advisory System and Benefits on the Intersection Level}

While TSP makes public transit more competitive, it results in significant delays to general traffic. In the near future, thanks to the development in intelligent transportation systems and the communication capabilities between vehicles and the infrastructure signals will be able to broadcast Signal Phase and Timing status information to nearby vehicles [18]. In 2014, Seredynski and Khadraoui [19] discussed two new complementary systems to the TSP. These systems are the Green Light Optimal Speed Advisory (GLOSA) and Green Light Optimal Dwell Time Advisory (GLODTA) systems. GLOSA provides a bus with speed guidance and GLODTA 
advices additional dwell time at the bus stop preceding the signals. The main target of these two systems is to allow buses to arrive at the intersection when the signal is green. The main advantage of these two systems is to improve the transit performance and on the same time do not modify the signal timing. Thus, unlike TSP, they do not create any additional delays to the general traffic. Results of Seredynski and Khadraoui [19] show that the two advisory systems provide the same level of benefits as the TSP system in terms of reductions in the number of stops and delay time at traffic signals. Additionally, results from field experiments show that when $50 \%$ of bus drivers follow the recommended speeds, the average bus speed increase significantly, while fuel consumption and emissions are reduced up to $20 \%$. Additionally, the use of these two systems does not have any negative effects on general traffic.

\section{Self-Regularization System (Buses Adapt to Signals and Signals Adapt to Buses)}

Based on the discussion in the previous section, it can be stated that in the future some objectives of TSP could be achieved by in-vehicle advisory systems or by taking the advantage of the vehicle connectivity and automation. Connectivity will facilitate the communication between buses and the infrastructure. This communication will allow signals to broadcast Signal Phase and Timing status information to nearby vehicles. Additionally, for the case of autonomous buses, speeds can be controlled to achieve a pre specific goal such as arriving at the intersection when the signal is green. Additionally, this new system can be complemented by the DTL to improve transit speed. As shown in the figure, system T3 is expected to enhance transit speed as the DTL will allow buses to travel near their free flow speed. Figure 4 shows a time space diagram example for three cases: T1: conventional TSP system, T2: conventional TSP complemented with speed the GLOSA and GLODTA, and T3: speed and dwell time advisory systems complemented with DTL. As shown in the figure, system T3 is expected to enhance transit speed as the DTL will allow buses to travel near their free flow speed.

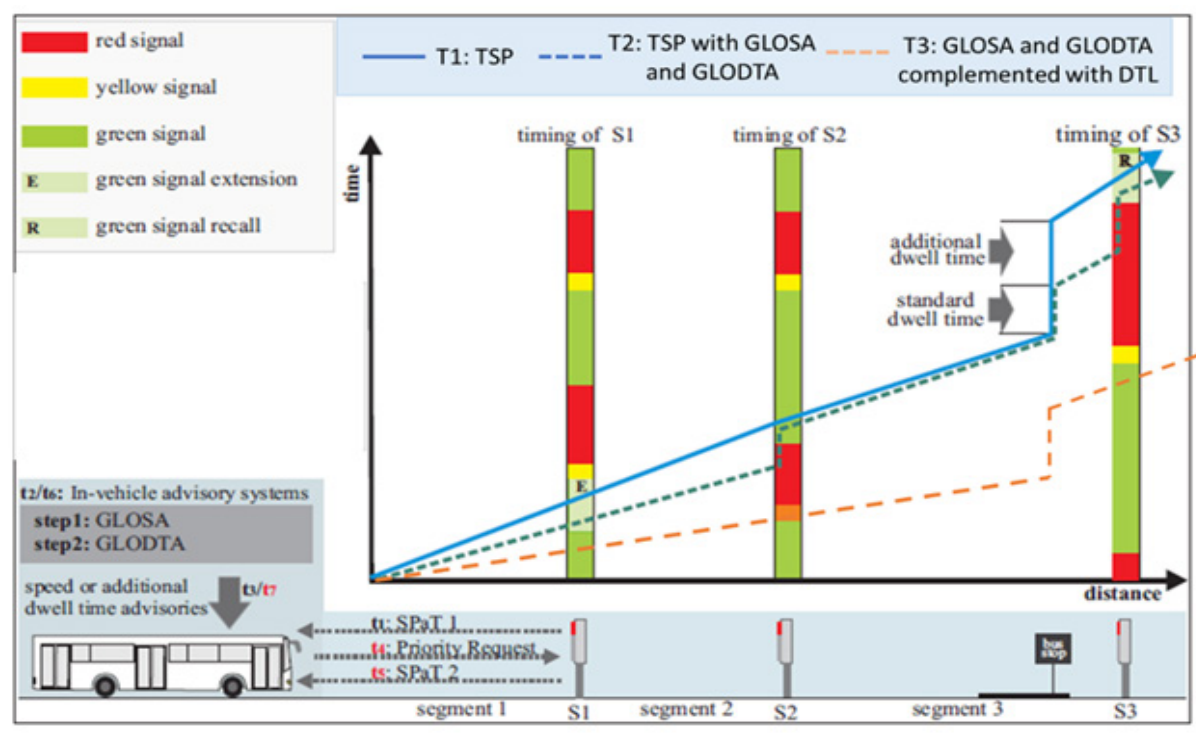

Figure 4: Overview of three cases (TSP, TSP with GLOSA and GLODTA) adopted from Seredynski and Khadraoui, 2014 [19].

\section{Conclusion}

It is expected that the introduction of autonomous vehicles will have a significant influence on the entire transportation system. However, there are growing consensus that AVs might worsen traffic and environmental conditions even if AVs use electric engines. Thus, some researchers have suggested that the introduction of AVs might not be environmentally friendly and that cities might need to prohibit the use of personal AVs and invest more in autonomous buses. This paper illustrates the benefits of the application of vehicle automation technology in the public transportation sector. Autonomous buses have the potential to provide many benefits such as:

1. Improve the quality of transit service: the use of the DTL allow buses to travel near the free flow travel speed which improve transit speed and in turn improve the quality of the service provided. Additionally, ABs have the potential to increase transit service frequency without significant increase in the overall costs as ABs reduce the operating costs significantly. Estimating transit service frequency is an optimization problem that balances the operating costs and passenger costs. Thus, ABs will allow for providing higher frequency with much lower operating costs, which will be very helpful for people in rural or devastated areas. This increase in the frequency will reduce passengers waiting time and will attract more users to transit.

2. Reduce the negative impact on traffic: it is widely stated that transit projects have adversarial impacts on traffic such as transit signal priority that provides priority to transit but increase traffic delay for the cross street. However, the use of 
the Self-regularization system that adjusts the bus speed to allow the bus to arrive at the intersection while the signal is green can improve the quality of transit service and on the same time does not increase the general traffic delay.

It must be mentioned that while many benefits can be achieved from the automation of buses, further research studies and focus from the academia are required to quantify the implications of the automation of public transportation service and understand how these implications will affect both transit agencies and transit users.

\section{Acknowledgement}

None.

\section{Conflict of Interest}

No conflict of interest.

\section{References}

1. SAFE (2018) The Economic and Social Value of Autonomous Vehicles: Implications from Past Network-Scale Investments.

2. KPMG (2015) Connected and Autonomous Vehicles - The UK Economic Opportunity.

3. Friedrich B (2016) The Effect of Autonomous Vehicles on Traffic Autonomous Driving pp.317-334.

4. Wagner P (2016) Traffic Control and Traffic Management in a Transportation System with Autonomous Vehicles. Autonomous Driving pp.301-316.

5. Brown A, Gonder J, Repac B (2014) An Analysis of Possible Energy Impacts of Automated Vehicle.

6. Miller SA, Heard BR (2016) The Environmental Impact of Autonomous Vehicles Depends on Adoption Patterns. Environ Sci Technol 50(12): 6119-6121.
7. Clark BY, Larco N, Mann RF (2017) The Impacts of Autonomous Vehicles and E-Commerce on Local Government Budgeting and Finance.

8. Metz D (2018) Developing Policy for Urban Autonomous Vehicles: Impact on Congestion. Urban Science 2(2).

9. Compass Transportation and Technology prepared for: Securing America's Future Energy (SAFE) (2018) The Economic and Social Value of Autonomous Vehicles: Implications from Past Network-Scale Investments.

10. Othman K (2020) Public Transportation on the Era of Autonomous Vehicles: Exploring Different Scenarios. Civil Eng Res J 10(5): 555800.

11. Gindrat R (2019) In a world of autonomous vehicles, this is why we'll need more public transport than ever. World Economic Forum.

12. SWI (2019) Driverless vehicles may lead to more congestion in cities.

13. Nagy V, Horváth B (2020) The effects of autonomous buses to vehicle scheduling system. The 11th International Conference on Ambient Systems, Networks and Technologies (ANT) Warsaw, Poland.

14.Viegas J, Lu B (2001) Widening the scope for bus priority with intermittent bus lanes. Transp Plan Technol 24(2): 87-110.

15. Viegas J, Lu B (2004) The intermittent bus lane signals setting within an area. Transp Res Part C Emerg Technol 12(6): 453-469.

16. Levin MW, Khani A (2018) Dynamic transit lanes for connected and autonomous vehicles. Public Transp 10: 399-426.

17. Currie G, Lai H (2008) Intermittent and dynamic transit lanes: Melbourne, Australia, experience. Transp Res Rec 2072: 49-56.

18. Seredynski M, Arnould G, Khadraoui D (2013) The emerging applications of intelligent vehicular networks for traffic efficiency. in Proc. 3rd ACM international symposium on Design and analysis of intelligent vehicular networks and applications pp. 101-108.

19. Seredynski M, Khadraoui D (2014) Complementing Transit Signal Priority with speed and dwell time extension advisories. 17th International IEEE Conference on Intelligent Transportation Systems (ITSC) Qingdao pp. 1009-1014. 